\title{
A Context Authentication Service for Role Based Access Control in Distributed Systems - CARDS
}

\author{
Ralph Holbein ${ }^{a}$ and Stephanie Teufel $b$ \\ a,b Department of Computer Science, University of Zurich \\ Winterthurerstr. 190, CH-8057 Zurich, Switzerland \\ email: \{holbein, teufel\}@ifi.unizh.ch
}

Information misuse is one of the major risks for information systems in organisations. Traditional approaches for authorisation and access control are insufficient because information misuse is primarily done by authorised people. These people have opportunity to access information even for unintended purposes. Role based access controls address this problem because access rights can be related to context descriptions (roles) and therefore, need-to-know access controls can be established. Need-to-know access controls define roles according to tasks in an organisation which represent intended purposes for information usage. Nonetheless, existing approaches for role based access controls do not ensure context authenticity during system operation, i.e. correspondence between activated roles and tasks within an organisation's actual business. Context authenticity must be ensured when a user activates a role or requests context related access to a particular object. Therefore, a context authentication service must be integrated with role based access controls. In this paper we describe the functionality and service components of a context authentication service called CARDS (Context Authentication Service for Role Based Access Control in Distributed Systems).

\section{INTRODUCTION}

Information is an asset for every organisation. Information systems (IS) in organisations are nowadays based on extensive use of information technology (IT). The permanently growing use of IT implies risks regarding information misuse. In this paper we first reason about the information misuse problem and subsequently, propose a security approach for information systems in organisations. The paper is structured as follows: In chapter 2 we introduce information misuse as an important security problem for information processing in organisations. We analyse this problem regarding characteristics of IT systems which increase the risk. In chapter 3, we introduce the need-to-know principle as a measure to prevent information misuse and role based access controls for implementation of need-to-know policies. Role based access controls provide means to specify context related access rights, i.e. access rights which depend on a user's context of activity. Need-to-know policies require to define the context of activity according to tasks in an organisation. We state the authenticity of a task-related access context during system operation as primary requirement to ensure a need-toknow, i.e. is a user's current context of activity really the task that he claims it is. We will demonstrate that existing approaches for role based access controls specify context related access rights, but do not provide means for authentication of a task-related context during system operation. Therefore, we introduce a context authentication service called CARDS in chapter 4 for integration with role based access controls. We explain the architecture and 
functionality of this security service as well as the integration of CARDS with role based access controls. Practicability of CARDS depends on realisation of a context notary. We refer to information systems in use which are suitable to realise this basic architectural component of CARDS. Finally, we give an example scenario to further illustrate how CARDS ensures intended rights of disposal for access to personal data. In this scenario CARDS is used to ensure privacy within police investigations.

\section{INFORMATION MISUSE: WHAT IS THE PROBLEM?}

Information misuse is a fundamental and unsolved problem for information security in organisations [12] [11]. Misuse of information means, that someone uses information for unintended purposes, i.e. neither intended nor acceptable by an owner of information or in other words an information source. It concerns organisational information as well as personal information where the demand for prevention of information misuse is even stated by law (privacy legislation) [34].

In order to develop adequate solutions it is fundamental to have a precise understanding of the problems which must be addressed. Security problems for that reason must be analysed in terms of risk analysis, i.e. what valuable goods must be protected, what are the relevant threats and where are the weak points that must be addressed? In the following paragraphs we will identify these issues regarding information misuse.

Information misuse is an undesirable side effect of information processing by humans, due to basic characteristics of human behaviour regarding utilisation and pragmatic impact of information within decision making [21]. Gurbaxani and Whang [8] for example discuss implications of information systems on organisations and markets. Their model of an organisation is quite useful to explain reasons for information misuse. An organisation is

".... a firm as an agency relationship built on a set of contracts among self-interested agents (employees). As a consequence, when decision-making authority is delegated to agents, it cannot be guaranteed that the decision will be aligned with the interest of the principal (shareholders). The divergence of interests can breed numerous problems and is costly to a firm (agency costs)." (p 60).

We state the self-interested behaviour of agents as a threat for information security in organisations. This threat is caused by authorised users of information systems and refers to their opportunity for intended as well as unintended usage of information that can be acquired from the accessible data. Therefore, we call this kind of threat opportunistic behaviour which is self-interested and guided by individual goals. Of course, the individual goals of an employee will usually overlap with the goals of the organisation, but will not be identical. Consequently, there is a difference in interests and goals. This difference is a threat for organisations and implies a risk to be damaged by opportunistic behaviour of employees.

Several studies during the last years confirm that the major threats for information security are not caused by unknown third party attackers but are caused by internal persons who are authorised and in confidence [35] [26] [2]. For that reason, we argue misuse of information is a prominent and serious security problem and must be addressed within the development of security controls which aim to protect information against usage for unintended purposes by authorised users of information systems.

The prominence of information misuse in the area of IT security even increases due to the fact that characteristics of IT systems contribute to this security problem, i.e. IT causes an information misuse risk. A risk depends on threats, assets and weak points. Unfortunately, there are basic characteristics of IT systems that support opportunistic behaviour and therefore, enable information misuse: 
- overall availability of data and system services for authorised users;

- easy copying of data.

From a security point of view, these characteristics are weak points to be damaged by opportunistic behaviour, i.e. vulnerable targets regarding information misuse. Obviously, this point of view results in conflicting goals between information security and desirable performance capabilities of IT systems. In the remainder of this paper we introduce a security approach which eliminates the previously described weak points and therefore, reduces the risk of information misuse.

\section{NEED-TO-KNOW POLICY}

A measure to protect against information misuse on a technical level is controlling data access according to the need-to-know principle. Data access is a precondition for acquisition of information [12]. This measure aims to address what we have previously called opportunistic behaviour. The goal is limitation of opportunity. A need-to-know policy treats data access rights as rights of disposal regarding information that may be acquired from the data, i.e. the pragmatic impact of data access is bound to intended purposes. Nonetheless, on a technical level the only way to restrict acquisition of information and therefore, to restrict pragmatic impacts of information, is to control data access [2]. Hence, need-to-know policies must be implemented by comprehensive data access controls.

\subsection{Objective and Limitation}

The need-to-know principle intends to restrict access rights to the smallest subset that is necessary to fulfil a user's actual task(s) in an organisation (also called least privileges). It is assumed that the less access rights a user has, the smaller is the risk of disclosure and manipulation and the more secure is a system [37]. However, a user may be responsible for several tasks within an organisation. Therefore, task-related access rights must be grouped for dynamic activation.

Certainly, prevention of information misuse by need-to-know access controls is limited. Need-to-know access controls reduce the information misuse risk because they ensure that an intended purpose exists when data access is granted. Nonetheless, they do not prevent information misuse at all because a user's overall access context is not restricted to his current task within an organisation. It is much more complex. Acquisition of information depends on multiple contingency factors, e.g. time, information processing capacity etc., and of course, the current knowledge of a user who acts within a social environment [21]. Consequently, the overall pragmatic impact of information cannot be determined and therefore, an information misuse risk still remains.

\subsection{Implementation of Need-to-Know Access Controls}

Commercial environments are characterised by the demand for decentralised and process oriented responsibilities as well as reduction of organisational hierarchies and dynamic changes of business [33] [28]. Therefore, access control mechanisms must provide flexible adaptation to changing needs. In general, mechanisms for implementation of need-to-know access controls must provide the following features:

1. Context related specification of access rights, i.e. access rights must be assigned to context descriptions (static). 
2. Assignment of users and context descriptions according to responsibilities for task fulfilment in an organisation (static).

3. Context authentication: context activation and control of access requests depending on an organisation's actual business (dynamic).

In the subsequent paragraphs we will further analyse these requirements and argue that role based access control mechanisms provide an appropriate foundation to implement need-toknow access controls. In section 3.3 we will further argue that current approaches for role based access controls do not provide for context authentication.

Role based access control mechanisms are technical means to implement need-to-know policies. First of all, we will briefly characterise role based access controls regarding traditional approaches [32] [17]: Identity based access controls define single access rights as discretionary relationship between a subject and an object (DAC: Discretionary Access Controls). Mandatory access controls (MAC) use global rules to combine static classifications of subjects and objects, i.e. capabilities and protection classes, but there are no discretionary relationships between subjects and objects. Identity based rights as well as static classification imply static rights for users because the users' identity is directly related to their access rights or capabilities, i.e. static rights will be assigned after successful user authentication.

Obviously, need-to-know access controls in organisations require some kind of dynamic classification, i.e. to change a user's access rights as the business proceeds. Moreover, the need for flexibility and process oriented responsibility in organisations as well as the fact, that commercial environments cannot be completely formalised and described as a closed system, require a mode of system operation where discretionary access controls with mandatory properties are best suited ${ }^{1}$ [31]. Therefore, we suggest role based approaches.

Role based access controls provide a discretionary specification of access rights as well as means to include mandatory properties: Access rights specify a discretionary relationship between a role and an object where roles are abstract descriptions of user classes (types of users) instead of identities, i.e. a role represents an access context. Consequently, multiple access rights for an object may refer to different roles and allow to define any number of different access contexts where an intended purpose for information usage exists. Consequently, users must be associated with a set of roles (static) according to their overall responsibility and may change these roles dynamically during system operation as the business proceeds, i.e. change their classification during system operation. Dynamic changes of user classifications are called role activation and deactivation. For that reason, roles must be defined according to the different tasks that users have to fulfil in an organisation and the assignment of users and roles must correspond to their responsibilities for task fulfilment. A task is a functional unit of work which results from a business and economic view on an organisation [27]. Roles can be used to represent such organisational tasks within the technical system, e.g. ProductShipment, OrderVerification, SW-Documentation etc. The assignment of responsible users defines who is allowed to activate which role(s), e.g. Smith is responsible for product shipment and Brown is responsible for order verification, product shipment as well as software documentation.

Certainly, there may be alternatives to role based, discretionary approaches. In [41] for example, the authors promote Multi Level Security (MLS) as an 'ideal' approach for Office Information Systems (OIS): "The multilevel secure system concept now being researched by industry for large-scale general-purpose or communication systems seems an ideal approach

1 In [31] the authors consider 'modes of operation' for secure systems as defined in the official UK glossary of computer security terminology. DAC will usually be applied if all users are basically allowed to have access to all information handled by the system but not all of them actually need to know all of the data. MACs are used when users need to have access to some of the data. 
for OIS if it can be designed for such environments." ( $p$ 88). However, we do not agree to that because MLS approaches are designed to represent formal systems. These approaches are not 'ideal' to implement need-to-know policies for commercial environments where process oriented responsibility and flexibility must be addressed ${ }^{2}$. Responsibility and flexibility depend on organisational design issues which include a notion of trust and confidence. Formal security models are not best suited to address these issues because an exact representation in terms of labels and capabilities with a mathematical order and relationships will usually be impossible. As a consequence, formal verification of security and security reasoning must not be the primary objective of a need-to-know policy implementation with role based access control mechanisms. In fact, in commercial environments it is not the primary objective.

In summary, we propose role based access controls where roles are subject classifications which represent the users' context of activity. A role is also an authorisation unit and provides a level of abstraction for a discretionary specification of authorisations. In order to realise a needto-know policy, roles must be defined according to the different tasks of users in organisations. Each authorised system user must be associated with a set of roles according to organisational responsibilities. Depending on his current task a user is allowed to activate/deactivate roles during system operation, i.e. activate/deactivate different sets of access rights [22].

\subsection{Deficiency of Current Approaches}

A prerequisite for every kind of access control is authentication of authorised system users. Role based approaches require additional authentication to handle the dynamic activation of rights after user authentication (activation and deactivation of roles) because roles build an intermediating layer between users and objects. A need to know depends on business activities which imply that particular tasks must be fulfilled, e.g. an order must be verified or shipment of an ordered product must be done. Therefore, need-to-know role activation must correspond to the organisation's current business transactions, i.e. the current tasks that must be fulfilled. This correspondence is called context authenticity and must be ensured by access controls. In other words, when a user activates a role, he states that he is responsible to fulfil a particular task which must actually be fulfilled due to the organisation's current business transactions and he needs the corresponding access rights now. Therefore, context authenticity concerns the question: "Is it true that a specified task must currently be fulfilled within the organisation?"

In general, context authenticity is of same importance as authenticity of the user himself! Otherwise, the need-to-know principle is not completely implemented. If a user can activate and change task-related roles without any control, there is no protection regarding information misuse, e.g. Brown activates the SW-Documentation role to access source code files and software documentation files for any purpose and whenever he wants without software documentation must be done.

There is a number of different approaches for role based access controls which aim to realise need-to-know policies. These approaches do not provide context authentication. We have selected several examples to point out this deficiency.

On a general level, the OSI Access Control Framework presents role definitions and context information to be used for access control [14]. The framework refers to context information like time periods, location etc. Task-related context definition for need-to-know access controls is not considered. The ECMA security model separates the context problem [3]: "Access Contexts are not considered here, and are for further studies" ( $p$ 39).

2 Of course, flexibility can be increased by trusted subjects which are allowed to change classifications or to violate write protection rules. However, this realises some kind of decentral authorisation but is not the original intention of MLS. 
Even on a much more concrete level where role based access control mechanisms have already been implemented the context authenticity problem is not addressed. Jonscher and Dittrich for example propose a very powerful mechanism [17]. System users must declare a role while they login the system. Later on, it is possible to change the active role during a user's activities. For this reason the system checks if the user is allowed to activate the selected role in general, i.e. verifies if the a corresponding user-role relationship is defined. Additionally, a matrix of conflicting roles is provided in order to prevent the simultaneous activation of particular role combinations, i.e. an undesirable accumulation of rights at a time. In [18] the authors remark that there is a need for specifying when and under what circumstances a user is allowed to activate a role. The access control mechanism provides predicates for that reason which can be used to control role activation, i.e. predicates must be valid when a role is activated. Further on, they use trigger mechanisms to repeat the validation of predicates after a role has been activated. Nonetheless, an application of this feature to ensure context authenticity regarding tasks within business transactions is not considered.

Lubinski proposes a "role-norm model" [24] which defines an information framework for every system user. Within this framework, the user is related to roles and responsible for a proper activation of his roles without additional controls. The same kind of user responsibility for role handling is used in the DORIS approach [10] which is an information system that takes privacy issues into account.

A system for personnel administration (VPS) is introduced in [10]. This approach includes working hours of an organisation to be used as additional access restriction. Again, task-related context authenticity is not considered, but the authors state the following requirement: appropriate procedures are necessary to prevent the unauthorised activation of roles! Further examples can be found in [16] [29] [38] [40].

In summary, current approaches consider static relationships between users and roles. Context authenticity as a dynamic property is neither ensured during role activation nor within single access requests. When a user 'logs into' (activates) one of his roles and states that a particular task must be fulfilled, the corresponding rights will be granted without further controls. Consequently, when a role is activated or an access request is granted, it cannot be guaranteed that a need to know really exists. Therefore, the need-to-know principle is reduced to specification of context related access rights (need-to-know administration of rights), but there is no need-to-know access control. For this reason information misuse is not addressed appropriately. Obviously, missing context authentication is a weak point regarding opportunistic behaviour of system users. These users are allowed to activate roles without any controls and can use the corresponding access rights whenever they want, even for unintended purposes.

Hence, a context authentication service which ensures that a need-to-know really exists is necessary to provide protection. This service may be used for context authentication during role activation but even within single access requests. According to the previous examples, as a precondition to activate the SW-Documentation role there must be a business transaction where a software documentation task must be accomplished. As a result, the context authentication service ensures that data access rights are treated as rights of disposal and bound to an intended usage of information. Nonetheless, the intention is the same as for user authentication: to ensure a correct representation of reality within the technical system [19] [6].

\section{CARDS}

In the previous chapter, we have explained that comprehensive implementation of the needto-know principle demands for context authentication within role based access controls. This is 
necessary to treat data access rights as rights of disposal with restriction to intended purposes regarding information usage. As a result, protection is no longer restricted to unauthorised access by third parties. There is additional protection against information misuse by authorised system users: overall availability of data is restricted to legitimate availability!

We have developed CARDS as a 'Context Authentication Service for Role Based Access Control in Distributed Systems' to provide context authentication within role based access controls. CARDS can be applied during role activation as well as to control single access requests. If CARDS is used to control single access requests there will be an add-on service called C-CARDS (Crypto CARDS) for encryption of data objects and further improvement of security. For that reason, CARDS was designed for distributed systems and integration in comprehensive distributed security architectures which provide basic services for authentication and trustworthy communication. CARDS aims to extend such environments, e.g. Kerberos authentication services [36], regarding implementation of need-to-know access controls. In the following sections we explain the general architecture and functionality of CARDS.

\subsection{Context Authentication during Role Activation}

Context authentication is based on a trusted instance that provides an authentication service called CARDS. This instance is the context notary $(\mathrm{CN})$ which realises a context authentication server (CAS). A CN registers an organisation's business activities in a context directory, i.e. keeps track of the business. Entries in the context directory represent tasks that must currently be fulfilled within business transactions. Therefore, these entries are dynamic and change as the business proceeds.

In general, context authentication is similar to user authentication procedures. The Directory Authentication Framework X.509 [15] for example, which is part of the X.500 directory service, describes different types of authentication procedures. The CARDS procedure is a single authentication as defined in X.509. Three types of principals are involved for context authentication: authenticated users, access control systems and the CAS. It is important to note, that user authentication is a precondition for context authentication. Authenticated users wish to obtain a particular set of access rights and therefore, declare a context of activity to the access control system ${ }^{3}$. This context must be authenticated by the $\mathrm{CN}$. The context authentication procedure is quite similar to well-known authentication services for distributed systems. Therefore, it is suitable for adaptation and integration with authentication services like Kerberos, KryptoKnight or SPX [6] [25] [19].

Nonetheless, there are also important differences to other authentication services. First of all, there is a difference concerning dynamic aspects of the registered items in the context directory. A directory for user authentication is quite static in contrast to a context directory. Entries of a context directory represent an organisation's business transactions and change as the business proceeds. Consequently, a context directory is dynamic in nature and its administration must be online with the progress of the business. Moreover, due to frequently changing entries context authentication must also be online. Another difference is that no secret information must be exchanged during context authentication, i.e. no confidentiality needs arise. In section 4.3 we will explain how context notary and administration of context directory entries can be realised in practice. First of all, we focus on the general architecture and functionality of the authentication service. Context authentication during role activation requires:

- integrity of messages passed,

- synchronisation of authentication messages to prevent replay,

- a trusted authentication server,

3 Declaration of a context of activity means the user states that a particular organisational task must be fulfilled right now. 
- management of the authentication server.

The following procedure is used for context authentication during role activation (Figure 1): A user selects a context descriptor $(\mathrm{Cx})$ or in other words declares the role he wants to activate and sends a role activation request to the access control system (1.), (2.). The access control system verifies authenticity of the specified context by the use of CARDS to request a context certificate from $\mathrm{CN}$, i.e. the context descriptor is passed to the $\mathrm{CN}$ (3.). The $\mathrm{CN}$ searches for a corresponding entry in the context directory to decide if the represented task is part of the current business transactions, i.e. must actually be fulfilled. If an entry was found, the context is valid and the $\mathrm{CN}$ generates a context certificate which essentially consists of the context descriptor, a time-stamp and a context validity flag 4 . The context certificate must be signed by asymmetric encryption with the private key of the context notary to prove origin and to guarantee integrity. Finally, the context certificate is passed back to the access control system for notification of the authentication result (4.). The access control system verifies time-stamp and signature and grants the context related access rights to the user in case of successful authentication because there is a need to know (5.). Otherwise role activation will be denied.

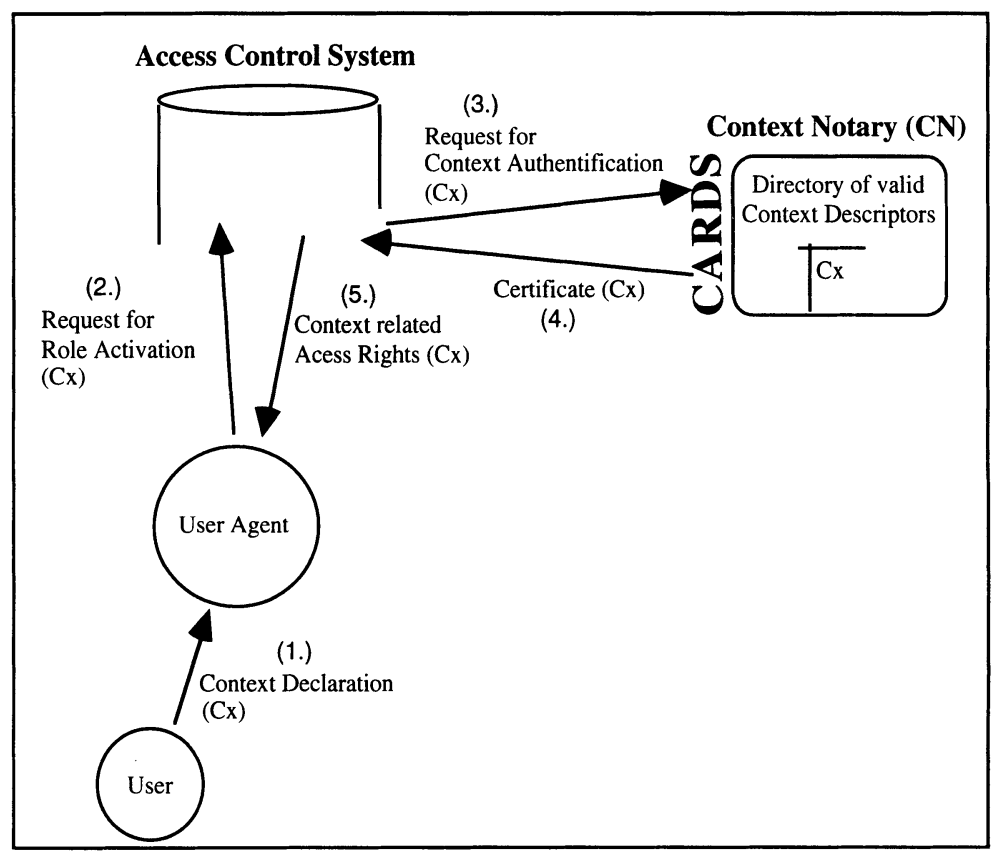

Fig. 1: Role activation with context authentication

4 Actually, we focus on a description of the general architecture and functionality of CARDS, therefore we do not specify the context certificate and the handling of its attributes in more detail. 


\subsection{Context Authentication and Data Encryption}

In the previous section we have explained context authentication during role activation. The same procedure can also be used to control single access requests. Moreover, it can be extended to further improve security for high sensitive data objects. This improvement is an add-on service to address the second weak point of IT as described in chapter 2: easy copying of data objects. The add-on provides capabilities for encryption and decryption of data objects and is called C-CARDS (Crypto-CARDS). Encrypted data objects prevent unauthorised copying and ensure that the access control system cannot be bypassed [41].

C-CARDS is provided by an object key notary $(\mathrm{OKN})$ as a second trusted instance. The $\mathrm{OKN}$ administrates cryptographic keys for encryption and decryption of data objects. Access to encrypted objects requires to obtain the corresponding object key from $\mathrm{OKN}$. In contrast to simple context authentication during role activation, this extension of the authentication service requires additional capabilities for confidential communication between the participating parties because object keys must be exchanged as well as data objects which are not encrypted. Hence, mutual authentication of communication partners as well as key distribution for confidentiality needs must provide for trustworthy communication apart from encrypting and decrypting the objects themselves. However, trustworthy protocol implementation is not considered in detail in this paper. It depends on embedding context authentication and object encryption in existing distributed security environments. In general, different approaches may be suitable. For that reason, we refer to well-known procedures and authentication services for example, prearranged secrets as used within Kerberos and KryptoKnight, certificates with long-term public and private keys as used within SELANE or even negotiation of secret keys by the communication partners themselves [6] [25] [19] [1].

Consequently, protection of objects with C-CARDS is quite extensive and therefore, its application must be selective, i.e. restricted to particular objects with high sensitivity regarding information misuse. For that reason, an object classification called CAR (Context Authentication Required) is used to declare protection needs regarding information misuse [4]. A CAR classification denotes that an object must be encrypted for storage as well as context authentication and decryption is necessary to access the data. Generation of and access to CARobjects requires trustworthy application programs which provide reliable functionality (no Trojan Horses etc.) and prevent external data flow, e.g. copying data into a clipboard etc. [5].

When a trustworthy application program generates a new CAR-object, CAR-classified data must be exchanged between this process and the access control system. As mentioned above, trustworthy communication must be initiated to prevent eavesdropping and interception. The trustworthy process encrypts the CAR-object and communicates the encrypted object to the access control system where it is decrypted again ${ }^{5}$. Now, the access control system uses CCARDS to request object key generation from OKN. The OKN generates an unique object key for symmetric encryption as well as an object identifier, signs these values together with a timestamp by asymmetric encryption with its private key and registers a new CAR-object in the object directory. Finally, OKN also initiates confidential communication with the access control system, encrypts signed object key and identifier and communicates the result to the access control system. The access control system decrypts object key and identifier and verifies timestamp and signature. Now the object key is used to encrypt the CAR-object for storage and the object identifier is registered for object administration and subsequent key requests. Finally, the access control system destroys the object key.

5 Additionally, the originating user must provide valid context descriptors and operations for initialisation of rights regarding the new CAR-object, i.e. task-related specification of intended purposes for information usage. Otherwise, some kind of object classification is necessary to associate a new object with a set of predefined generic rights [17] [18]. Specification of task-related rights for CAR-objects is considered in [13]. 
Hence, stored CAR-objects are encrypted and can only be accessed via the access control system which is authorised to use C-CARDS in order to obtain object keys from the OKN. Therefore, acces $\$$ tickets must be provided for C-CARDS. An access ticket consists of a context certificate and a CAR-object identifier. As context certificates must be generated by CARDS, context authentication is a precondition for the provision of object keys.

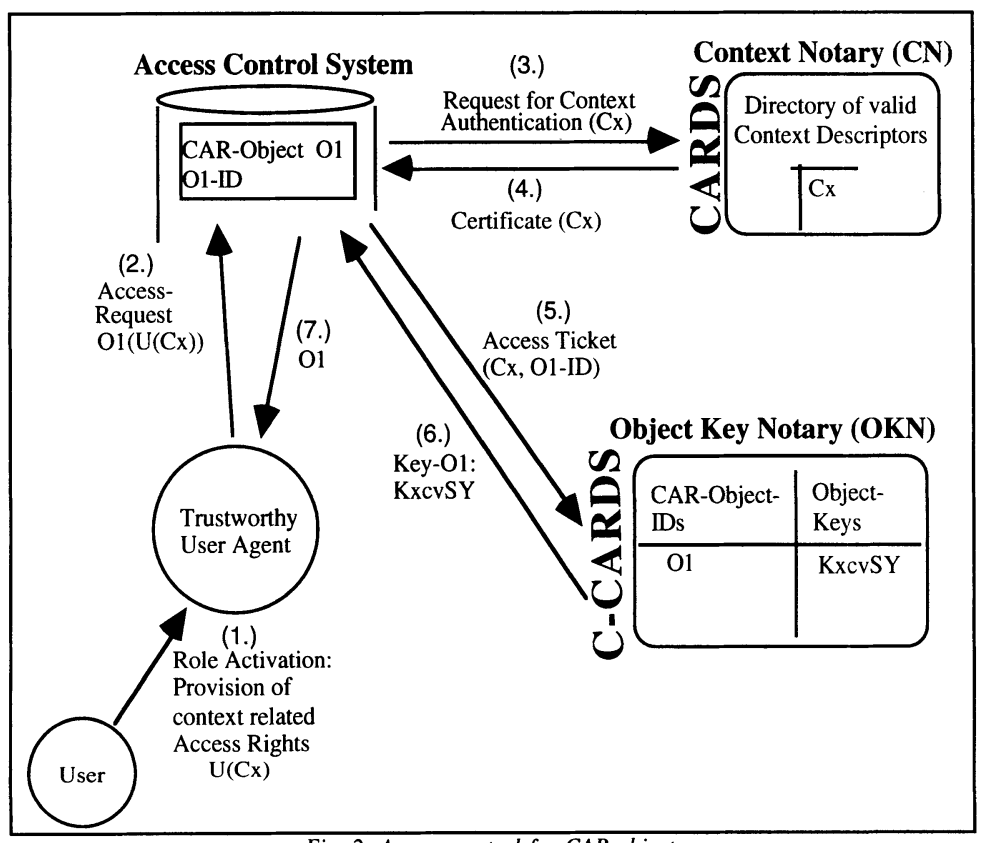

Fig. 2: Access control for CAR-objects

The following procedure controls access to CAR-objects (Figure 2) ${ }^{6}$ : As already mentioned, access of a user (U) to a CAR-object $(\mathrm{O} 1)$ must be mediated by a trustworthy application program (Trustworthy User Agent) which acts on behalf of that user. This process initiates trustworthy communication with the access control system within an access request (2.). We assume, there was a prior role activation enforced by the access control system and therefore, a role has already been activated for the user $(\mathrm{U}(\mathrm{Cx}))$ (1.). Further on, we assume this role represents a valid access context regarding the CAR-object O1. Now the access control system uses CARDS to request a context certificate from the $\mathrm{CN}$ in the same way as explained for role activation in the previous section ${ }^{7}$ : The context descriptor of the user's activated role is passed

6 The diagram includes the essential parts of context authentication and object encryption but does not include authentication of communication partners as well as key distribution and encryption-decryption for communication between the access control system and the trustworthy application program as well as the object key notary.

7 Now, CARDS is used to verify authenticity of a context (role) which has already been activated. In case of selective context authentication on object access level there is not necessarily a context authentication during role activation. Nonetheless, if context authentication has also been done during role activation, there will be a re-authentication for single access requests. 
to the context notary (3.). Subsequently, the context notary verifies if the context is valid according to the registrations in the context directory. Again, if context authentication is successful, the context notary generates a context certificate with a time stamp (4.). The context certificate is used by the access control system to generate an access ticket (Cx, O1-ID) which proves that there are valid access conditions at a particular time. The access ticket is used to call C-CARDS in order to request the corresponding object key from OKN (5.). Now, the OKN searches for the object identifier in the object key directory, selects the corresponding object key and communicates the object key (Key-O1: KxcvSY) to the access control system (6.). Again, this communication must be trustworthy and is done in the same way as previously described for object key generation. The access control system is now able to decrypt the CAR-object. Finally, the decrypted object is communicated to the requesting user agent (7.) using the same protection as described for CAR-object generation. This procedure ensures intended purposes for access to CAR-objects, because access must be directed via the access control system where context authentication is enforced.

\subsection{Service Provision}

We will now demonstrate that a realisation of the previously described security services is practicable in today's organisations. The basic component or provider of the authentication service is a context notary which administrates entries in a context directory. Practicability of the CARDS services depends on the realisation of the context notary. Several established technologies and information systems in use are suitable for a realisation of the context notary. These systems are used for different kinds of process control in organisations. The basic requirement for implementation of the context notary is control of business activities. Business activities are directly related to tasks within an organisation and therefore, can be mapped to task-related role definitions of an access control system. In the following, we propose three types of IT-systems in organisations which control business activities and therefore, will be suitable to provide CARDS:

- Workflow management systems control business processes. A business process consists of structured activities which directly represent tasks that must be fulfilled when the business proceeds. Business process descriptions provide highly structured and very detailed task specifications which map task-related role definitions of an access control system. Therefore, workflow management systems are suitable to provide context authentication with high granularity: single activities in workflows can be used for role definitions and context authentication.

- Time accounting and billing systems control working hours of employees. The working hours are directly related to business activities for billing and invoicing of customers or cost centres. Time accounting and billing systems provide project numbers to control semi-structured business activities which can be mapped to task-related role definitions of an access control system. Therefore, time accounting and billing systems are suitable to provide context authentication with medium granularity: activities in a project environment, e.g. project numbers according to different project phases, can be used for role definitions and context authentication.

- Delegation and auditing systems control ad-hoc activation of tasks within an organisation. Delegation results in activated tasks that must be actually fulfilled by responsibility holders. Multiple types of tasks and granularity may be defined for delegation. Therefore, delegation and auditing systems are suitable to provide context authentication with arbitrary granularity depending on the task definitions: irregular but predefined activities which depend on external events can be used for role definitions and context authentication. 
Workflow management systems are used on different levels of an organisation [9]: on a strategic level there are topics like Lean Management, Business Process Redesign, Organisation Redesign, Innovation; on a tactical level it is modelling, analysis and re-organisation of core process parts, and on the operational level there is improvement of processes and control during system operation within daily business. Due to the growing importance of workflow management systems for business control and the fact that these systems directly map business activities, tasks and role definitions, we propose workflow systems to be most practicable for a realisation of the context notary and the provision of CARDS. There is a large number of workflow applications in different fields of industry and administration [7, 20, 23, 30]. Currently, there are systems like ActionWorkflow ${ }^{\mathrm{TM}}$, HyperFlow ${ }^{\mathrm{TM}}$, FlowMark ${ }^{\mathrm{TM}}$ etc. in practical use providing potential for comprehensive integration of organisation and information processing [9]. Additional, integration of security services has important implications regarding reliability and administration of workflow systems. Provision of CARDS requires reliable functionality concerning business process control and security administration to prevent fraudulent manipulations, e.g. modifying or pretending business transactions. Nonetheless, security of workflow systems is for further consideration but not addressed in this paper.

\section{APPLICATION SCENARIO}

Finally, we explain an application scenario which illustrates the relevance of a context authentication service to ensure privacy of personal data within governmental authorities. This example can easily be adapted to a broad range of governmental authorities where privacy legislation requires to prevent misuse of personal information, e.g. police departments, refugee authorities, social welfare office, public health department etc.

It is important to note, that our scenario refers to some basic characteristics of German and Swiss privacy legislation. There are governmental privacy officers who register each personal database used in organisations. Moreover, collections of personal data must be declared regarding intended rights of disposal which bind the usage of information to particular purposes. For example, German law concerning central electronic registration of foreign people requires that the purpose information will be used for must be declared as a precondition for automated access to personal data [39].

We have selected police investigations for further illustration of the scenario (Figure 3$)^{8}$ : Police authorities administrate several databases with personal data referring to criminals, witnesses, observed persons etc. The legal usage of each personal data object is bound to predefined, intended purposes. Therefore, these objects must be classified as CAR-objects and associated with particular tasks concerning police investigations. The context notary is realised by a workflow management system for coordination and control of police investigations ${ }^{9}$. This system is classified as context notary with security administration and is installed at a police department site. Further on, a central object key notary provides C-CARDS and is installed at the governmental privacy officer's site. Access to personal data can now be controlled as explained in section 4.2 for single access requests: Roles and role based access rights to the objects within the 'Observed Persons' database are defined according to tasks within police investigations. The workflow system provides CARDS for generation of context certificates (3.), (4.) when access to the 'Observed Persons' database is requested. The location of the object key notary ensures that every access request for personal data is routed via the governmental

8 The authentication procedure is restricted to the essential interactions. Therefore, arrows (1.), (2.) and (7.) from Fig. 2 were omitted.

9 Probably this will not be possible for every kind of investigation. 
privacy officer's site (5.), (6.). Access tickets which will be passed to C-CARDS can be collected for later revision of police investigations and validation of legality by the privacy officer.

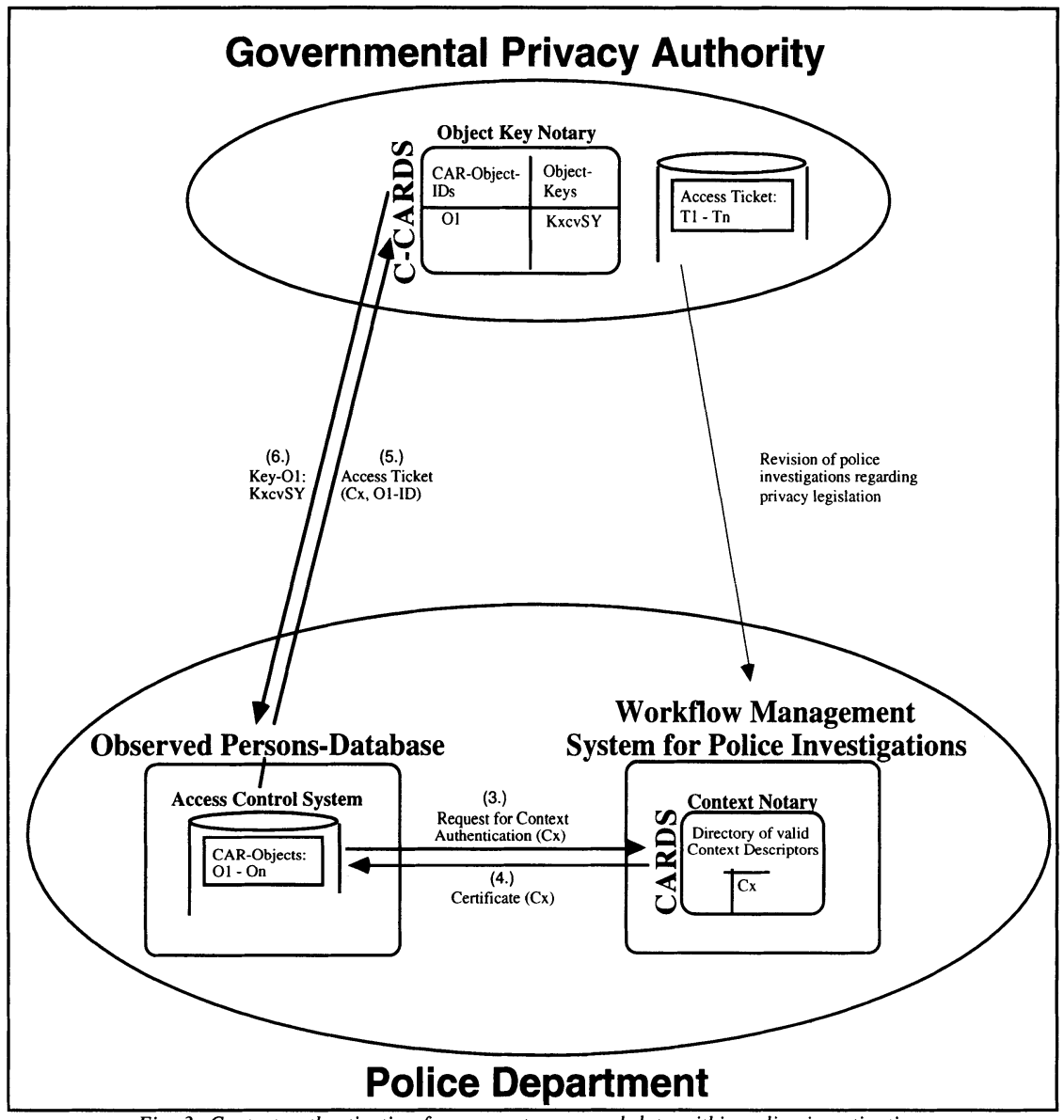

Fig. 3: Context authentication for access to personal data within police investigations

\section{CONCLUSIONS AND FURTHER WORK}

In this paper we have addressed an important risk regarding information security in organisations: information misuse. In a first part we have analysed reasons for information misuse and explained the weak points of IT-systems which must be protected. In a second part the need-to-know principle was proposed as a measure to address the information misuse problem and requirements were formulated for an implementation of need-to-know access controls. Role based access control mechanisms were proposed to implement need-to-know 
access controls. We have demonstrates that current approaches for role based access controls lack of capabilities for ensuring context authenticity. In a third part, we have addressed this deficiency and introduced the context authentication service CARDS for integration in distributed security architectures. This service enhances role based access controls to realise need-to-know policies. Moreover, the context authentication service can be extended by the crypto service C-CARDS for further improvement of security regarding unauthorised copying of data objects and bypassing the access control system.

The CARDS approach shows the need for integration of computer security controls with an organisation's business in order to achieve effective information security in commercial environments. The focus of this paper was to introduce the CARDS idea. Therefore, we have explained the general architecture and functionality of the service. In order to demonstrate its practicability we have referred to existing IT-systems in organisations which are suitable to realise the central context notary.

Of course, further work must be done for a more detailed specification of the authentication procedure, the mechanisms required for implementation of the services and their integration in existing security architectures. Moreover, the service access points must be specified to integrate CARDS with role based access controls. Currently, we prepare for a prototype implementation of CARDS based on ActionWorkflow ${ }^{\mathrm{TM}}$ workflow management.

\section{REFERENCES}

[1] T. Beth, D. Gollmann, Security Systems Based on Exponentation Primitives. Will be published in Proceedings of IFIP TC11 Tenth International Conference on Information Security SEC'94, Curacao, Dutch Caribbean, Elsevier Science Publishers.

[2] J. Biskup, Sicherheit in Informationssystemen - Gewährleistung und Begrenzung des Informationsflusses. GI-Fachtagung Verlässliche Informationssysteme VIS'91, Darmstadt, Springer Verlag, 1991, pp. 1-21.

[3] ECMA, Security in Open Systems - A Security Framework. 46, European Computer Manufacturers Association (ECMA), 1988.

[4] J.H.P. Eloff, R. Holbein, S. Teufel, Security Classification for Documents. Submitted for publication in Computers\&Security, 1994.

[5] D. Fox, M. Böttger, SECUBOOT - Authentisches remote boot für Client-ServerNetzwerke. Fachtagung Sicherheit in Informationssystemen, Zürich, vdf Verlag, 1994, pp. 161-173.

[6] D. Gollmann, T. Beth, F. Damm, Authentication Services in Distributed Systems. Computer \& Security, vol. 12/93, 1993, pp. 753-764.

[7] R. Grell, Schriftgutverwaltung und Vorgangsbearbeitung in der Landesverwaltung Baden-Württemberg. Theorie und Praxis der Wirtschaftsinformatik HMD, vol. 31, no. 176, 1994, pp. 35-44.

[8] V. Gurbaxani, S. Whang, The Impact of Information Systems on Organizations and Markets. Communications of the ACM, vol. 34, no. 1, 1991, pp. 59-73.

[9] H. Heilmann, Workflow Management: Integration von Organisation und Informationsverarbeitung. Theorie und Praxis der Wirtschaftsinformatik HMD, vol. 31, no. 176, 1994, pp. 8-21.

[10] S. Herda, S. Mund, A. Steinacker (eds.), Szenarien zur Sicherheit informationstechnischer Systeme. München, R. Oldenbourg Verlag GmbH, 1993.

[11] H.F. Hofmann, R. Holbein, Reaching out for Quality: Considering Security Requirements in the Design of Information Systems. International Conference on 
Advanced Information System Engineering CAiSE*94, Utrecht, Netherlands, 1994, pp. 105-118.

[12] R. Holbein, Secure Information Exchange in Organisations. Will be published in Proceedings of IFIP TC11 Tenth International Conference on Information Security SEC'94, Curacao, Elsevier North Holland.

[13] R. Holbein, S. Teufel, A Security Design Method for Information Exchange in and between Organisations. Proceedings Priority Programme Informatics Research, Information Conference Module 1, Secure distributed system, Bern, Switzerland, 1994, pp. $1-10$

[14] ISO, ISO 10181-3: Information technology - Open Systems Interconnection - Security frameworks in open systems - Part 3: Access Control. ISO/IEC DIS 10181-3, International Organisation for Standardization ISO, 1991.

[15] ISO/CCITT, The Directory - Authentication Framework X.509. ISO 9594-8; X.509, Organisation for International Standardization ISO, 1988.

[16] J.I. Jones, M. Sergot, Formal Specification of Security Requirements using the Theory of Normative Positions. Computer Security - ESORICS 92, Toulouse, France, Springer Verlag, 1992, pp. 103-121.

[17] D Jonscher, K.R. Dittrich, A Formal Security Model Based on an Object-Oriented Data Model. 93.41, University of Zurich, Department of Computer Science, 1993.

[18] D. Jonscher, K.R. Dittrich, Realisierung von Sicherheitsstrategien mit Hilfe flexibler Zugriffskontrollmechanismen. Fachtung Sicherheit in Informationssystemen, Zürich, vdf Verlag, 1994, pp. 23-52.

[19] S. Kanungo, Identity Authentication in Heterogenous Computing Environments: A Comparative Study for an Integrated Framework. Computers \& Security, vol. 13 no. 3, 1994, pp. 231-253.

[20] M. Kübler, Elektronische Vorgangsbearbeitung in der Beschaffung. Theorie und Praxis der Wirtschaftsinformatik HMD, vol. 31, no. 176, 1994, pp. 58-67.

[21] R. Kuhlen, The pragmatic added-value of information. Language games with basic concepts from information science. Report 1/89, University of Constance, 1989.

[22] L.G. Lawrence, The Role of Roles. Computers \& Security, vol. 12/93, 1993, pp. 15-21.

[23] U. Leisten, K. Bandat, Workflow Management in der Software-Entwicklung. Theorie und Praxis der Wirtschaftsinformatik HMD, vol. 31, no. 176, 1994, pp. 68-79.

[24] A. Lubinski, Ein Rollen-Normen-Modell für den konzeptionellen Entwurf von Sicherheitsanforderungen in Unternehmensinformationssystemen. GI-Fachtagung Verlässliche Informationssysteme VIS 93, München, Vieweg Verlag, 1993, pp. 67-84.

[25] R. Molva, G. Tsudik, E. Van Herreweghen et al., KryptoKnight Authentication and Key Distribution System. Proceedings of Computer Security - ESORICS 92, Toulouse, France, Springer Verlag, 1992, pp. 155-174.

[26] P. Neumann, Trusted Systems. In: K.M. Jackson, J. Hruska and D.B. Parker (eds.), Computer Security Reference Book Oxford, Butterworth-Heinemann Ltd, 1992, pp. 837-862.

[27] H. Österle, R. Saxer, T. Hüttenhain, Organisatorisches Monitoring in der Gestaltung von Geschäftsprozessen. Wirtschaftsinformatik, vol. 36 , no. 5, 1994, pp. 465-477.

[28] A. Picot, Restrukturierung von Unternehmen und Beschäftigungsperspektiven. Office Management, vol. 11/94, 1994, pp. 10-14.

[29] F. Rabitti, E. Bertino, W. Kim et al., A Model of Authorization for Next-Generation Database Systems. ACM Transactions on Database Systems, vol. 16, no. 1, 1991, pp. 88-131.

[30] H. Reinermann, Vorgangssteuerung in Behörden. Theorie und Praxis der Wirtschaftsinformatik HMD, vol. 31 , no. 176, 1994, pp. 22-34. 
[31] C.L. Robinson, S.R. Wiseman, A Consideration of the Modes of Operation for Secure Systems. Proceedings of Computer Security - ESORICS 94, Third European Symposium on Research in Computer Security, Brighton, United Kingdom, Springer Verlag, 1994, pp. 335-356.

[32] R.S. Sandhu, Lattice-Based Access Control Models. Computer (IEEE), vol. 26, no.11, 1993, pp. 9-19.

[33] M. S. Scott Morton (eds.), The corporation of the 1990's. Information technology and organizational change. New York/Oxford, Oxford University Press, 1991.

[34] H.J. Smith, Privacy Policies and Practices: Inside the Organisational Maze. Communications of the ACM, vol. 36, no. 12, Business Computing, 1993, pp. 105-122.

[35] M. Smith, Document Security. In: K.M. Jackson, J. Hruska and D.B. Parker (eds.), Computer Security Reference Book Oxford, Butterworth-Heinemann Ltd, 1992, pp. 333-347.

[36] J.G. Steiner, C. Newman, J.I. Schiller, Kerberos: An Authentication Service for Open Network Systems. UNIX Security Workshop USENIX, Portland, OR, 1988.

[37] G. Steinke, M. Jarke, Support for Security Modeling in Information Systems Design. IFIP WG 11.3 Workshop on Database Security, VI: Status and Prospects, Vancouver, Canada, Elsevier Science Publishers B.V., 1992, pp. 125-141.

[38] H. Strack, K.-Y. Lam, Context-Dependent Access Control in Distributed Systems. IFIP/SEC93, 9th International Computer Security Symposium and Exhibition, Toronto, Kanada, Elsevier Science Publishers, 1993.

[39] C. Streit, Datenschutzregelungen des Ausländerzentralregistergesetzes. Datenschutz und Datensicherung DuD, vol. 10/94, 1994, pp. 559-568.

[40] T.C. Ting, S. Demurjian, M.-Y. Hu, Requirements, Capabilities, and Functionalities of User-Role Based Security for an Object-Oriented Design Model. IFIP WG 11.3 Workshop on Database Security 1991, Sheperdstown, West Virgina, Elsevier Science Publishers, 1992, pp. 275-296.

[41] R.B. Vaughn Jr., H. Saiedian, E.A. Unger, A Survey of Security Issues in Office Computation and the Application of Secure Computing Models to Office Systems. Computer \& Security, vol. 12 no.1, 1993, pp. 79-97. 